\title{
BMJ Open International experiments with different models of allocating funds to facilitate integrated care: a scoping review protocol
}

\author{
Akram Khayatzadeh-Mahani, ${ }^{1,2}$ Ellen Nolte, ${ }^{3}$ Jason Sutherland, ${ }^{4}$ \\ Pierre-Gerlier Forest ${ }^{1}$
}

To cite: Khayatzadeh-Mahani A, Nolte $\mathrm{E}$, Sutherland $\mathrm{J}$, et al. International experiments with different models of allocating funds to facilitate integrated care: a scoping review protocol. BMJ Open 2018;8:e21374. doi:10.1136/ bmjopen-2017-021374

\section{- Prepublication history and} additional material for this paper are available online. To view these files, please visit the journal online (http://dx.doi. org/10.1136/bmjopen-2017021374).

Received 28 December 2017 Revised 21 June 2018 Accepted 12 September 2018

Check for updates

(C) Author(s) (or their employer(s)) 2018. Re-use permitted under CC BY-NC. No commercial re-use. See rights and permissions. Published by BMJ.

For numbered affiliations see end of article.

Correspondence to

Professor Pierre-Gerlier Forest; pgforest@ucalgary.ca

\section{ABSTRACT}

Introduction Integrated care is viewed widely as a potential solution to some of the major challenges faced by health and social care systems, such as those posed by service duplication, fragmentation and poor care coordination, and associated impacts on the quality and cost of services. Fragmented models of allocating funds to and across sectors, programmes and providers are frequently cited as a major barrier to integration and countries have experimented with different models of allocating funds to enhance care coordination among service providers and to reduce ineffective care and avoid costly adverse events. This scoping review aims to assess published international experiences of different models of allocating funds to facilitate integration and the evidence on their impacts.

Methods and analysis We will adopt a scoping review methodology due to the potentially vast and multidisciplinary nature of the literature on different models of allocating funds in health and social care systems, as well as the scarcity of existing knowledge syntheses. The framework developed by Arksey and 0'Malley will be followed that entails six steps: (1) identifying the research question(s), (2) searching for relevant studies, (3) selecting studies, (4) charting the data, (5) collating, summarising and reporting the results and (6) and conducting consultation exercises. These steps will be conducted iteratively and reflexively, making adjustments and repetitions when appropriate to make sure the literature has been covered as comprehensively as possible. To ensure comprehensiveness of our literature review, we also search a wide range of sources.

Ethics and dissemination An integrated knowledge translation strategy will be pursued by engaging our knowledge users through all stages of the review. We will organise two workshops or policy roundtables/policy dialogues in Alberta and British Columbia with participation of diverse knowledge users to discuss and interpret the findings of our review and to draw out policy opportunities and lessons that can be applied to the context of these two provinces.

\section{INTRODUCTION}

There is a growing policy emphasis on the integration of care within the health sector

\section{Strengths and limitations of this study}

- This review will employ a broad search strategy that includes both peer-reviewed literature and grey literature.

- This review will adopt an integrative approach to knowledge translation through engaging diverse knowledge users in design, analysis and dissemination of findings.

- The quality of evidence or grading evidence, that are part of systematic reviews, will not be assessed in this review as in other scoping reviews.

- By limiting the search language to English, we may miss some potentially important and relevant findings.

- The scope of this review is very broad. As an example, integrated care per se has been used as an umbrella term for various concepts and organisational structures. This may lead to uncovering an extensive literature that could appear unmanageable.

and between the health and other sectors, mainly social care, aiming to ensure that people receive the right care, at the right time and in the right place. ${ }^{1}$ Integrated care (IC) is viewed widely as a potential solution to overcome some of the major challenges that health and social care systems are facing. ${ }^{12}$ It is considered as an approach for addressing financial and quality issues through tackling duplication, fragmentation and poor care coordination. ${ }^{3}$ WHO has shifted emphasis to IC to achieve universal health coverage and ensure high-quality and cost-effective service delivery. ${ }^{3} 4$ Some potential impacts of IC include: improved access to care; enhanced experience and satisfaction for patients, carers and healthcare providers; reduced secondary care utilisation; improved quality of life and health status; improved health outcomes; reduced unnecessary duplication of care and improved cost-effectiveness. ${ }^{5-9}$ 
IC has been used as an umbrella term for various concepts and organisational structures. ${ }^{10}$ There is a plethora of concepts/terminologies used such as, among others, 'IC', 'coordinated care', 'collaborative care', 'continuity of care', 'managed care', 'disease management' and 'case management', which reflects the diversity of objectives behind adopting these concepts and a variety of disciplines that have applied this concept. ${ }^{711} \mathrm{It}$ is suggested that in defining IC, the emphasis should be placed on the needs of services users, their families and the communities to which they belong instead of structures and organisations. ${ }^{10}$ Indeed, there is considerable supportive evidence highlighting that such a perspective should be the heart of any IC strategy in order to bring together potentially competing factions in a unifying narrative. ${ }^{12}$ With this consideration, IC has been widely defined in the context of improving quality and access to care especially for people with complex, long-term health problems whose needs cut across multiple providers, services and settings. ${ }^{1} 1013$

Fragmented models of allocating funds to and across sectors, programmes and providers are frequently cited as major barriers for the implementation of IC. ${ }^{914-16}$ In this review, we use the terminology of 'allocating funds' by adopting the "world health report $2000{ }^{17}$ framework on health system performance, which classifies allocation of funds as a key component of healthcare financing. Healthcare financing deals with three basic functions of revenue collection, pooling of resources and resource allocation and purchasing. Revenue collection deals with how health systems raise money from different sources (eg, households, businesses and external sources). Pooling refers to the accumulation and management of revenues for the common advantage of participants ${ }^{18}$ so members of the pool share collective health risks. ${ }^{19}$ Resource allocation and purchasing refers to the methods employed to purchase services from public and private providers, a process through which revenues collected in fund pools are allocated to institutional or individual providers for delivering health services and interventions. ${ }^{18}$ In this review, our focus is on allocation of resources/funds to and across sectors, programmes and providers. We will look at the micro and mesolevel of resource allocation (eg, allocating funds to individual healthcare providers and hospitals) and the macrolevel resource allocation in terms allocating funds to and across sectors (healthcare vs social care or long-term care), service/programme areas or scope of care (eg, prevention, acute care, rehabilitation, palliative care), population groups (eg, elder care, persons with disabilities) and health conditions (eg, diabetes, joint replacement). We will also search for the laws, legislations and Acts that countries have enacted to facilitate IC through allocation of funds to and across sectors, programmes and providers.

Since traditional models of allocating funds such as fee-for-service do not financially incentivise integration of care ${ }^{20}$ countries are increasingly experimenting with new forms of allocating funds (especially macrolevel models) to incentivise care coordination and integration. ${ }^{14}$ Examples include episode-based bundled payments and population-based integrated payment methods. Bundled payments are single payments to groups of providers involved in providing a defined episode of care for a particular health condition (eg, diabetes) with the aim of strengthening an integrated approach to service delivery. ${ }^{21}$ Example of bundled model is the Bundled Payments for Care Improvement Initiative in USA. ${ }^{22}$ Under the population-based integrated payment methods, rooted in the global capitation models adopted by Health Maintenance Organizations in USA in 1980s and 1990s, groups of providers are funded for managing care of a defined population. Here, a group of providers share accountability for costs and quality of care for a segment of population. Two prominent examples of these models include Accountable Care Act Accountable Care Organizations in $\mathrm{USA}^{23}$ and Gesundes Kinzigtal model in Germany. ${ }^{24}$

Countries have also enacted laws, legislations and Acts to facilitate IC through pooling of resources across sectors. For example, in England, the Health and Social Care Act 2012 aimed, among others, to promote a closer integration of services across sectors and the Care Act 2014 tasked local authorities with promoting the integration of care between health and health-related services, like housing, with the aim of increasing patient experience of care and improving quality of care. ${ }^{25}$ In Germany, the 2015 Health Care Strengthening Act promotes IC through a number of measures such as establishment of an 'innovation fund' totalling $€ 300$ million annually for start-up funding of innovative IC programmes. ${ }^{26}$ Despite these legislations, barriers to IC including resources levels, differing status related to knowledge and expertise, value differences, lack of role clarity, stereotyping and competitiveness, and clash of professional cultures remain. ${ }^{2728}$

Unfortunately, existing literature provides only limited information on synthesis of diverse models of allocating funds to facilitate integration that countries have adopted and the evidence on their impacts. In this review, we will search for these models. The review's first goal is to facilitate an analysis of the diverse models of allocating funds that countries have experimented with to enhance care coordination and integration and the evidence on their impacts. The review's second goal is to describe the context of the models, so that the contexts can be contrasted with those in Canada. Moreover, the historical basis for models of allocating funds will add 'colour' to the context(s). The review's third goal is to synthesise these findings into policy opportunities and lessons learnt aiming to draw out approaches and methods that can be applied to the Canadian context with a focus on Alberta and British Columbia provinces.

To increase the uptake of our review findings, we will engage diverse knowledge users including content experts, policy and decision-makers, and community organisations in the design, analysis and dissemination 
of the review. In this review, we are going to address the following objectives: (1) to map, analyse and synthesise existing knowledge and the main sources and types of evidence about different models of allocating funds to facilitate integration, (2) to develop a conceptual framework that classifies those models, (3) to explore different policy objectives behind adopting/developing those models, (4) to investigate potential barriers and facilitators to implementation of diverse models, (5) to explore the impact and degree of success for those models where the degree of success is measured against the outcomes that health systems are trying to achieve including, among others, care integration, cost growth reduction and maximisation of patients' clinical and experience outcomes, (6) to identify additional gaps in the literature and (7) to draw out policy opportunities and lessons learnt that can be applied to the Canadian context with a focus on Alberta and British Columbia provinces.

\section{METHODS AND ANALYSIS}

Scoping reviews systematically map the key concepts within a research area and the main sources and types of evidence available through a comprehensive review of the literature. ${ }^{29-31}$ They are different from systematic reviews in two distinctive ways: (1) a systematic review typically focuses on a well-defined question and includes specific study designs identified a priori while a scoping review addresses a broader topic and includes many different study designs. (2) A systematic review tends to answer a very specific and narrow research question and assesses the quality of studies for inclusion while a scoping review tends to answer to a broader research question and does not assess the quality of studies for inclusion. ${ }^{31}$ A scoping review can inform a systematic review. ${ }^{32} 33$

Given the vast nature of the literature on IC and allocation of funds (both within the health system and across health and other sectors), and thus far limited efforts to synthesise existing knowledge, we will adopt a scoping review method. The scoping review also assists in providing greater conceptual clarity about how the literature has addressed a complex and wide topic. ${ }^{34}$ It can also help determine the value of undertaking a full systematic review on this topic. ${ }^{33}$ We have conceived our review as a method in its own right that will lead to the publication and dissemination of research findings on models of allocating funds to facilitate IC. Synthesis of existing evidence and consultation of findings with a wide range of stakeholders will allow us to draw out policy opportunities and lessons that can be applied to the Canadian context with a focus on Alberta and British Columbia provinces. Although we will identify gaps in the existing evidence that may lead to a full systematic review, we are not aiming to conduct a systematic review.

In this scoping review, we will follow the framework developed by Arksey and O'Malley which has been further updated by Levac et $a l^{30}$ This framework entails six steps: (1) identifying the research question/s, (2) searching for relevant studies, (3) selecting studies, (4) charting the data, (5) collating, summarising and reporting the results and (6) and conducting consultation exercises. These steps are treated in an iterative way and we will engage with each step in a reflexive way and repeat steps, where necessary, to make sure that the literature is comprehensively covered. ${ }^{29}{ }^{31}$ To ensure comprehensiveness of our literature review, we also search a wide range of sources.

\section{Step 1: identifying the research question/s}

As the focus of scoping reviews is on summarising the breadth of evidence, the research questions should be broad. ${ }^{31}$ A well-defined research question at the first step linked with a clear purpose helps later steps of the review including study selection and data extraction. ${ }^{30}$ The overarching question that guides our review is: "what is the range of models of allocating funds to facilitate integration that have been documented in the published and grey literature?' We initially generated a list of potential research questions based on our research team experience and initial engagement with the relevant literature. We then consulted with our knowledge users, including content experts and policy and decision-makers, via email to seek their views on the research questions and to refine and finalise them. This input from knowledge users and ongoing engagement with them will ensure the study's rigour, relevance and comprehensiveness. This ongoing engagement, in turn, will lead to greater potential for the review results to be taken up by a broad range of knowledge users. ${ }^{35}$ The following research questions will guide this review:

1. What is the range of existing models of allocating funds to facilitate care integration that have been documented in the published, unpublished and grey literature and what problems were these models trying to address?

2. What is the range of policy objectives driving the development or adoption of identified models?

3. What are the barriers to and facilitators of implementation for models of allocating funds to facilitate care integration in the context of the problem trying to be solved?

4. What is the evidence of impact of identified models of allocating funds to facilitate care integration as given by authors?

5. How do funders of, and knowledge users involved in, this scoping review evaluate evidence of impact of new funding models? Or what are the outcome measures they highly value and expect to be impacted by the new funding models?

6 . What is the evidence on the degree of success of identified models of allocating funds to facilitate care integration where the degree of success is measured against the outcomes that health systems are trying to achieve including, among others, care integration, cost growth reduction and maximisation of patients' clinical and experience outcomes? 
7. What are the policy opportunities and lessons that Canada can learn from identified models of allocating funds to facilitate IC?

\section{Step 2: identifying relevant studies}

At this step, we will identify relevant studies and will develop a search strategy, terms/concepts to use, sources to be searched, time span and language. ${ }^{31}$ As Arksey and O'Malley's recommend that 'comprehensiveness is the whole point of scoping the field, ${ }^{31}$ we will employ a very broad search strategy. We will use a search strategy worksheet ${ }^{36}$ and our search terms will include keywords related to (1) allocation of funds AND (2) integration of care. We will adjust search terms based on nuances of each database. Our key concepts will include, but not be limited to (1) allocation of funds, (2) IC and (3) healthcare (see online supplementary appendix table 1 for our detailed search strategy and terms). We will refine our search terms and perform more sensitive literature searches throughout the review process, as necessary. We will undertake the following five activities as part of the broad search strategy: electronic database search, web search, handsearch of relevant journals, citations of relevant papers and scanning the reference lists of relevant papers.

We will employ an information scientist (or library scientist) to perform the electronic database search. Given the multidisciplinary nature of the research project we will be using diverse electronic databases including: Web of Science, PubMed, MEDLINE via Ovid; EMBASE via Ovid excluding MEDLINE; Cumulative Index to Nursing and Allied Health Literature; Applied Social Science Index and Abstract; Health Management Information Consortium; EconLit; Sociological Abstracts; Social Science Citation Index; Scopus, Cochrane Library and PsycINFO. Google Scholar and Google will be also searched for published and grey literature (see the full list of databases in online supplementary appendix table 2 ). We conducted a preliminary search in MEDLINE, which produced 8668 records (20 November 2017).

We will search the following websites for unpublished and grey literature: OpenGrey; Dissertations \& Theses A\&II via ProQuest; ISI Proceedings; Conference Proceedings Citation Index-Social Science and Humanities; Joanna Briggs and ProQuest Dissertations and Theses; PAIS Index-Public Affairs Information Service; Google Scholar and Google. We will also search the website of key institutions and organisations such as WHO, WHO Europe, International Foundation for Integrated Care, the European Observatory on Health Systems and Policies, Agency for Healthcare Research and Quality, National Institute for Health and Care Excellence, National Institutes for Health and Canadian Institutes of Health Research (CIHR). The research team and knowledge users will identify other websites and sources at the review progresses.

Once these electronic searches were completed, we will perform a handsearch of key journals (eg, The
International Journal of Integrated Care; Health Policy; Health and Social Care in the Community; Health Policy and Planning; Journal of Health Services Research and Policy; Health Services Research; Social Policy and Administration; BMC Health Services Research; The BMJ; Critical Social Policy; Plos One; Health Affairs; The New England Journal of Medicine; JAMA) which will be identified by the research team and content experts. We will also track citations of relevant papers. Finally, we will search the reference lists of relevant papers to find papers not identified in our initial search. We will import all retrieved searches into EndNote $\mathrm{X} 8$ in which the duplicate references will be identified and discarded. We anticipate a manual search for duplicates as selected electronic databases to download citations and referencing are often inconsistent in their content and formatting. ${ }^{35}$

\section{Step 3: relevance testing}

We will adopt a team approach, which increases the rigour of our review, ${ }^{30}$ to determine which studies/materials to include. The team will discuss and finalise the inclusion and exclusion criteria at the beginning of the scoping review. The research team has initially decided on the following inclusion and exclusion criteria. However, given the unclear boundaries of scoping reviews at the outset, predefined inclusion and exclusion criteria are provisional and may be revised and refined following further engagement with our knowledge user partners and with emerging knowledge of the existing literature. ${ }^{37}$

\section{Initial inclusion criteria}

- Papers that discuss models of allocating funds to facilitate IC.

- Published or unpublished primary studies (quantitative, qualitative or mixed-methods studies), theses/ dissertations, conference papers, theoretical discussions and grey literature.

\section{Initial exclusion criteria}

- Papers published before 2000.

- Papers not published in English.

- Book reviews.

- Commentary, opinion pieces, editorial papers and descriptive papers that provide no relevant empirical evidence.

Studies and materials will be included through a two-step process. First, all abstracts and executive summaries will be scanned by two independent reviewers. Then, the same reviewers will retrieve all potentially relevant full papers and materials for inclusion consideration. Following Levac et $a l^{30}$ recommendation, our reviewers will meet at the start, middle and end of the abstract review process in order to discuss any challenges or ambiguities related to study selection and to refine, where necessary, the search strategy. A scanning tool will be developed, in consultation with our knowledge user partners, to determine the relevance of papers to IC funding models and also to code the type of data retrieved (eg, reviews, theoretical discussion, 
empirical data, government documents, policy brief, web content, conference paper). Two graduate students, who have received training in the scoping review process, will be recruited to screen the titles, abstracts/summaries or executive summaries that are yielded from the search strategy for study selection. Records will be classified by these reviewers as 'potentially relevant' or 'exclude'. When the relevance of a publication is in doubt, they will retrieve the full text. To make sure the selection process in non-biased, two members of the research team will independently review $1 \%$ of the abstracts/summaries and compare their results with the graduate students' results. Our research questions may require some refinement at this stage to ensure the review is feasible and relevant without compromising the comprehensiveness of the search.

In the second step, the graduate student reviewers will independently retrieve and review all full texts coded as 'potentially relevant' as part of considering them for inclusion. If there are disagreements between the two reviewers on inclusion, the other team members will be consulted to make the final decision. The research team will organise monthly meetings/teleconferences during this stage to discuss findings, progress, challenges and uncertainties related to study selection.

\section{Step 4: charting the data}

We will extract contextual or process oriented data from the included studies using a narrative descriptive synthesising approach. ${ }^{30}{ }^{31}$ We will use a deductively generated coding tree and import the data into NVivo V.10 for data analysis. Our research team will collectively develop the data-charting form (or extraction form), using Microsoft Excel sheets, to determine which variables to extract that best help answer our research questions. The data-charting/extraction form will be derived from our research questions and also from the best relevant papers. The charting will be treated as an iterative process in which we will constantly update the data-charting form as the analysis proceeds, ${ }^{30}$ similar to the process used in inductive coding in qualitative data analysis. The two graduate students with two members of the research team will independently extract data from the first five studies, using a data-charting form, to check if their data extraction approach is consistent with the research questions and objectives. The graduate students will then independently continue extracting. To ensure accuracy and completeness the research lead will double check the extracted data. The data extracted will include: countries/ locations, author/s or institution/s or organisation/s, publication title, publication year, research question or study purpose or policy goal/s, type of funding models, barriers/facilitators to implementation of models and evidence of success/impact, if available. As the research team becomes more familiar with the literature, this list of extracted data will be modified.

\section{Step 5: collating, summarising and reporting the results}

This step generally constitutes the most extensive phase of a scoping review. After extracting all data we will establish a working group to meaningfully interpret the data. With the research questions in mind, the two graduate students will quantify the extracted data and produce a descriptive summary of the included materials (eg, for journal papers, we will extract overall number of studies included, types of study design, year of publication, countries/locations where studies were conducted and type of integrated funding models).

The analytical synthesis of extracted data is critical in scoping reviews as these reviews are not a short summary of journal papers and grey literature. We will conduct a constant comparative analysis using NVivo V.10 in order to organise our data into overarching categories. Constant comparison analysis allows comparisons to be made across concepts, similarities, differences, and gaps to be identified, and a conceptual framework to emerge. During the synthesis phase, we will systematically combine the extracted data and will develop a taxonomy of models of allocating funds to facilitate integration. A conceptual framework will be developed with the following key elements which will be the starting point for our coding nodes too: the funding models, barriers and facilitators, and policy success/impact. At this phase, we will solicit the views of our knowledge users via email, teleconference or web-conference to allow their feedback and inputs in reviewing the findings, before we can provide policy recommendations. ${ }^{31}$

\section{Step 6: Consultation}

Consultation enhances the methodological rigour of the review as well as validity of the study outcome and should be a compulsory stage in scoping reviews. ${ }^{30}$ In our scoping review, we will engage knowledge users at all steps of the review by which we will move beyond knowledge translation towards an iterative integrated knowledge translation (iKT).$^{38}$ We will seek knowledge users' input for a number of reasons, among others, to further refine the review questions; to tailor our review findings to the knowledge users' needs; to add a higher level of meaning, content and expertise to our review preliminary findings and to make our review findings more applicable. Our knowledge users will be engaged in the first steps of the review via email and teleconference. A workshop will be held with knowledge users to have their inputs/feedbacks for developing the comprehensive conceptual framework that classifies integrated funding models.

\section{PATIENT AND PUBLIC INVOLVEMENT}

In line with the CIHR Strategy for Patient Outcome Research definition of patient engagement in health research, our patient engagement plan will incorporate patients as respected and active partners in the research process and we aim to engage 'patients' meaningfully in all stages of our scoping review. In our research, we have adopted the broad definition of 'patient', not just the person who receives care, but any person or group 
with lived experience of a health or health systems issue, including caregivers and family members.

We are working with two organisations in Alberta and British Columbia that provide our access to 'patient' groups. IMAGINE Citizens, which is an independent group of Alberta citizens who participate in patient-oriented research, is our point of access to various 'patient' groups in Alberta. British Columbia Primary Health Care Research Network (BC-PHCRN) is also our access point to 'patients' in BC. In writing this scoping review protocol, we have shared our proposal, including the research questions, with IMAGINE Citizens and BC-PHCRN for their inputs and feedback and have incorporated them into the protocol. We will engage 'patients' at all steps of our review, towards an iterative iKT format.

\section{ETHICS AND DISSEMINATION}

The aim of this scoping review is to synthesise the existing literature on diverse models of allocating funds to and across sectors, providers and programmes that countries have experimented with to enhance care coordination and integration and the evidence on their impacts, to enhance understanding about these models and to extrapolate policy recommendations that may be particularly relevant to the Canadian context with a focus on Alberta and British Columbia provinces. We anticipate this knowledge synthesis will provide a number of key outputs, most importantly: (1) a conceptual framework that classifies models of allocating funds to facilitate integration, (2) potential barriers and facilitators for implementing those models.

On completion of the review, we will disseminate the results via diverse means (see online supplementary appendix table 3 for full list of dissemination tools for different target audiences). We will present the findings at academic conferences and publish a research report as well as two academic peer-reviewed papers. The comprehensive conceptual framework that classifies models of allocating funds to facilitate integration will be made freely available online as an evidence repository. We will further publish a series of policy brief, developed in collaboration with our knowledge user partners about how to promote and better implement a funding model that facilitates care integration through use of findings of this review. Other means to disseminate our review results include blogs that intersect academic and popular internet dissemination; a webinar in collaboration with our knowledge users; a short (4-5 min) YouTube (or series of YouTube videos) discussing policy implications of the findings and media interviews to disseminate findings and support their uptake. An iKT strategy will be pursued as our knowledge users are closely engaged throughout the entire research cycle, and directly contribute to the policy-relevant publications of the project.

\section{Author affiliations}

${ }^{1}$ School of Public Policy, University of Calgary, Calgary, Alberta, Canada
${ }^{2}$ Health Services Management Research Center, Institute for Futures Studies in Health, Kerman University of Medical Sciences, Kerman, Iran

${ }^{3}$ Department of Health Services Research and Policy, Faculty of Public Health and Policy, London School of Hygiene \& Tropical Medicine, United Kingdom

${ }^{4}$ Centre for Health Services and Policy Research, School of Population and Public Health, University of British Columbia, Vancouver, Canada

Contributors AK-M and P-GF conceived the review approach. AK-M wrote the first draft of the manuscript. P-GF, EN and JS critically reviewed and revised the manuscript. All authors read and approved the final version of the manuscript.

Funding This study was supported by Canadian Institutes of Health Research (ClHR).

Competing interests None declared.

Patient consent Not required.

Provenance and peer review Not commissioned; externally peer reviewed.

Open access This is an open access article distributed in accordance with the Creative Commons Attribution Non Commercial (CC BY-NC 4.0) license, which permits others to distribute, remix, adapt, build upon this work non-commercially, and license their derivative works on different terms, provided the original work is properly cited, appropriate credit is given, any changes made indicated, and the use is non-commercial. See: http://creativecommons.org/licenses/by-nc/4.0/.

\section{REFERENCES}

1. Goddard M, Mason AR. Integrated care: a pill for all ills? Int $J$ Health Policy Manag 2016;6:1-3.

2. Kodner DL. All together now: a conceptual exploration of integrated care. Healthc Q 2009;13 Spec No:6-15.

3. Lê G, Morgan R, Bestall J, et al. Can service integration work for universal health coverage? Evidence from around the globe. Health Policy 2016;120:406-19.

4. World Health Organization. WHO global strategy on people-centred and integrated health services: interim report. 2015.

5. Exworthy M, Powell M, Glasby J. The governance of integrated health and social care in England since 2010: great expectations not met once again? Health Policy 2017;121:1124-30.

6. Curry N, Ham C. Clinical and service integration. The route to improve outcomes London: The Kings Fund, 2010.

7. Nolte E, Pitchforth E. What is the evidence on the economic impacts of integrated care? 2014.

8. Erens B, Wistow G, Mounier-Jack S, et al. Early evaluation of the integrated care and support pioneers programme. 2016.

9. Mason A, Goddard MK, Weatherly HLA. Financial mechanisms for integrating funds for health and social care: an evidence review. 2014.

10. Kodner DL, Spreeuwenberg C. Integrated care: meaning, logic, applications, and implications-a discussion paper. Int J Integr Care 2002;2:e12.

11. Nolte E, McKee M. Integration and chronic care: a review. Caring for people with chronic conditions $A$ health system perspective 2008:64-91.

12. Ham C, Walsh N. Making integrated care happen at scale and pace. 2013.

13. Struckmann V, Leijten FRM, van Ginneken E, et al. Relevant models and elements of integrated care for multi-morbidity: Results of a scoping review. Health Policy 2018;122:23-35.

14. Nolte E. Financing and Reimbursement. In: Handbook Integrated Care: Springer, 2017:165-87.

15. Cameron A, Lart R, Bostock L, et al. Factors that promote and hinder joint and integrated working between health and social care services: a review of research literature. Health Soc Care Community 2014;22:225-33.

16. Hultberg EL, Glendinning $C$, Allebeck $P$, et al. Using pooled budgets to integrate health and welfare services: a comparison of experiments in England and Sweden. Health Soc Care Community 2005;13:531-41.

17. Daher M. Overview of the world health report 2000 health systems: improving performance. J Med Liban 2001;49:22-4.

18. Murray CJ, Frenk J. A framework for assessing the performance of health systems. Bull World Health Organ 2000;78:717-31.

19. Schieber G, Baeza C, Kress D, et al. Financing Health Systems in the 21st Century. Disease control priorities in developing countries 2006:225-42. 
20. Tsiachristas A. Financial incentives to stimulate integration of care. Int J Integr Care 2016;16:8.

21. Sutherland J, Hellsten E. Integrated funding: connecting the silos for the healthcare we need. 2017.

22. Centers for Medicare \& Medicaid Services Bundled Payments for Care Improvement (BPCl). Initiative: general information. 2017.

23. Fisher ES, Shortell SM. Accountable care organizations: accountable for what, to whom, and how. JAMA 2010;304:1715-6.

24. Hildebrandt H, Hermann C, Knittel R, et al. Gesundes Kinzigtal integrated care: improving population health by a shared health gain approach and a shared savings contract. Int $J$ Integr Care 2010;10:1-14.

25. Humphries R. Integrated health and social care in England-Progress and prospects. Health Policy 2015;119:856-9.

26. Milstein R, Blankart CR. The health care strengthening act: the next level of integrated care in Germany. Health Policy 2016;120:445-51.

27. Cameron A. What have we learnt about joint working between health and social care? Public Money \& Management 2016;36:7-14.

28. Cameron A. Impermeable boundaries? Developments in professional and inter-professional practice. J Interprof Care 2011;25:53-8.

29. Daudt HM, van Mossel C, Scott SJ. Enhancing the scoping study methodology: a large, inter-professional team's experience with
Arksey and O'Malley's framework. BMC Med Res Methodol 2013;13:48.

30. Levac D, Colquhoun H, O'Brien KK. Scoping studies: advancing the methodology. Implement Sci 2010;5:69.

31. Arksey H, O'Malley L. Scoping studies: towards a methodological framework. Int J Soc Res Methodol 2005;8:19-32.

32. Armstrong R, Hall BJ, Doyle J, et al. 'Scoping the scope' of a cochrane review. J Public Health 2011;33:147-50.

33. Langlois EV, Ranson MK, Bärnighausen T, et al. Advancing the field of health systems research synthesis. Syst Rev 2015;4:90.

34. Davis K, Drey N, Gould D. What are scoping studies? A review of the nursing literature. Int J Nurs Stud 2009;46:1386-400.

35. Valaitis R, Martin-Misener R, Wong ST, et al. Methods, strategies and technologies used to conduct a scoping literature review of collaboration between primary care and public health. Prim Health Care Res Dev 2012;13:219-36.

36. Riedling AM. Learning to learn: a guide to becoming information literate: ERIC, 2002.

37. Adom T, Puoane T, De Villiers A, et al. Protocol for a scoping review of existing policies on the prevention and control of obesity across countries in Africa. BMJ Open 2017;7:e013541.

38. Grimshaw J. A Knowledge Synthesis Chapter: Canadian Institutes of Health Research, 2015. 